\title{
Systematic Review \\ Relative Effect of Extracorporeal Shockwave Therapy Alone or in Combination with Noninjective Treatments on Pain and Physical Function in Knee Osteoarthritis: A Network Meta-Analysis of Randomized Controlled Trials
}

\author{
Chun-De Liao ${ }^{1,2} \mathbb{D}^{\mathrm{D}}$, Yu-Yun Huang ${ }^{3}$, Hung-Chou Chen ${ }^{2,4} \mathbb{D}$, Tsan-Hon Liou ${ }^{2,4} \mathbb{D}$, Che-Li Lin $^{5,6,+}$ \\ and Shih-Wei Huang $2,4, *,+\mathbb{D}$
}

1 Master Program in Long-Term Care, College of Nursing, Taipei Medical University, Taipei 110301, Taiwan; 08415@s.tmu.edu.tw

2 Department of Physical Medicine and Rehabilitation, Shuang Ho Hospital, Taipei Medical University, New Taipei City 235041, Taiwan; 10462@s.tmu.edu.tw (H.-C.C.); peter_liou@s.tmu.edu.tw (T.-H.L.)

3 Department of Pediatrics, New York University Langone Medical Center, New York, NY 10016, USA; huang-yu-yun@hotmail.com

4 Department of Physical Medicine and Rehabilitation, School of Medicine, College of Medicine, Taipei Medical University, Taipei 110301, Taiwan

check for

updates

Citation: Liao, C.-D.; Huang, Y.-Y.; Chen, H.-C.; Liou, T.-H.; Lin, C.-L.; Huang, S.-W. Relative Effect of Extracorporeal Shockwave Therapy Alone or in Combination with Noninjective Treatments on Pain and Physical Function in Knee Osteoarthritis: A Network Meta-Analysis of Randomized Controlled Trials. Biomedicines 2022, 10, 306. https://doi.org/10.3390/ biomedicines 10020306

Academic Editors:

Chang-Chun Hsiao and Yao-Chi Chuang

Received: 25 December 2021

Accepted: 24 January 2022

Published: 28 January 2022

Publisher's Note: MDPI stays neutral with regard to jurisdictional claims in published maps and institutional affiliations.

Copyright: (C) 2022 by the authors. Licensee MDPI, Basel, Switzerland. This article is an open access article distributed under the terms and conditions of the Creative Commons Attribution (CC BY) license (https:// creativecommons.org/licenses/by/ $4.0 /)$.
5 Department of Orthopedic Surgery, Shuang Ho Hospital, Taipei Medical University, New Taipei City 23561, Taiwan; 11010@s.tmu.edu.tw

6 Department of Orthopedics, School of Medicine, College of Medicine, Taipei Medical University, Taipei 11031, Taiwan

* Correspondence: 13001@s.tmu.edu.tw; Tel.: +886-2-2249-0088 (ext. 1600)

† Shih-Wei Huang and Che-Li Lin contributed equally to this study.

\begin{abstract}
Extracorporeal shockwave therapy (ESWT) has been recommended for managing pain in patients with knee osteoarthritis (KOA). The difference in therapeutic effects between radial shockwave characteristics (RaSW) and focused shockwave characteristics (FoSW) with different energy levels for KOA remains controversial. The purpose of this network meta-analysis (NMA) was to identify the effects relative to the different ESWT regime and combination treatments on pain and functional outcomes in individuals with KOA. The randomized controlled trials (RCTs) which investigated the efficacy of RaSW, FoSW, and combination treatments in patients with KOA were identified by searches of electronic databases. The included RCTs were analyzed through NMA and risk-of-bias assessment. We analyzed 69 RCTs with a total of 21 treatment arms in the NMA. Medium-energy FoSW plus physical therapy, medium-energy acupoint RaSW plus Chinese medicine, and high-energy FoSW alone were the most effective treatments for reducing pain [standard mean difference $(\mathrm{SMD})=-4.51$ ], restoring function $(\mathrm{SMD}=4.97)$, and decreasing joint inflammation (SMD $=-5.01)$. Population area and study quality influenced the treatment outcomes, particularly pain. Our findings indicate that medium-energy ESWT combined with physical therapy or Chinese medicine is beneficial for treating pain and increasing function in adults with KOA.
\end{abstract}

Keywords: osteoarthritis; shockwave therapy; pain; function; inflammation

\section{Introduction}

Knee osteoarthritis (KOA) is a serious joint disease and prevalent chronic musculoskeletal disorder, with pain being its primary symptom and main clinical presentation [1,2]. In KOA, knee pain occurs at an early stage of the disease and is gradually aggravated throughout disease progression [3]. With disease progression, KOA impairs musculoskeletal system [4], ultimately leading to physical difficulty [2,5,6]. Such musculoskeletal pain in KOA is closely associated with a decline in health state and a negatively 
impact on quality of life [7-9]. In addition, the most common practical problem of KOA is the pain-induced limitation in physical function and mobility, especially walking ability [10-12], stair negotiation [13], and postural transition in activities [10-12]. Under such circumstances, the development of efficient treatment strategies for pain management is essential for individuals with KOA.

Extracorporeal shockwave therapy (ESWT) is a convenient, cost-effective treatment for managing pain in common musculoskeletal conditions of the lower limbs [14-18]. ESWT is safely used in clinical practice and serves as an alternative to conservative injections or surgery owing to its noninvasive and effective application in musculoskeletal disorders $[19,20]$. In addition, ESWT could play a role in regenerative medicine through its stimulation of soft tissue healing [21-23] and cartilage regeneration [24,25] and inhibition of pain receptors [26]. On the basis of the sources of energy production and the delivery pathway that propagates acoustic energy through biological tissue, ESWT can be characterized into two types, namely focused shockwave (FoSW) and radial shockwave (RaSW) [27-29]. FoSW and RaSW use different energy sources to generative shockwave impulses [29-33], and each type of two ESWT applications should be served as an independent treatment modality $[29,31,32]$. The effects of ESWT depend on the energy level which is presented as energy flux density (EFD, $\mathrm{mJ} / \mathrm{mm}^{2}$ ) per shockwave impulse [28,30]. The energy level of ESWT ranges from 0.001 to $0.5 \mathrm{~mJ} / \mathrm{mm}^{2}$ [30,31,34,35]. Identifying relative effects among different shockwave applications is important due to that an overly high dosage may have high risks of poor treatment outcome and adverse events [36].

A number of systemic review and meta-analysis studies have investigated the efficacy of ESWT in the treatment of KOA [37-43]. Most trials included in these reviews and meta-analysis studies conducted an ESWT intervention in combination with noninjection treatments such as conventional physical therapy (CPT) and traditional Chinese medicine (TCM). However, few of the systemic reviews compared ESWT with other interventions [40-43]. The relative effects of the various combination treatment regimens composed of different ESWT applications (i.e., FoSW versus RaSW and low versus high EFD) and noninjective treatments, with reference to usual care (UC), have yet to be determined. In addition, all of these systemic reviews investigated the effects of ESWT on perceived pain and function outcomes [37-43], among which only two reported the combined effects on joint range of motion and walking performance [41,43]; none focused on the disease inflammation outcomes of arthritic knees. Therefore, in this study, the effects of ESWT on physical mobility, joint function, and disease inflammation were investigated in individuals with KOA.

The relative effects among different combination treatment regimens of various ESWT applications remain unclear. Therefore, the purpose of this study was (1) to identify the relative effects of different ESWT applications and combination treatment regimens on pain outcome, global function, and disease inflammation through network meta-analysis (NMA) and (2) to determine the optimal treatment strategy by using the ranking probabilities of each intervention type for individuals with KOA.

\section{Materials and Methods}

\subsection{Study Design}

The present NMA study was conducted in accordance with the guidelines of the Preferred Reporting Items for Systematic Reviews and Meta-Analyses Statement (PRISMA) [44] and the additional statement for NMA [45,46]. A comprehensive electronic search of online sources was performed to identify eligible randomized controlled trials (RCTs) reporting the efficacy of ESWT for KOA. All of the articles were identified from electronic databases, including the Physiotherapy Evidence Database (PEDro), PubMed, Cochrane Library Database, Embase, China Knowledge Resource Integrated Database, and Google Scholar. In addition, secondary sources included trials enrolled in previous systemic reviews retrieved from the aforementioned sources. 
There was no limitation of the language or publication year, which minimized language and publication bias. At the beginning of search, all potentially eligible articles were independently searched by two team members, CDL and SWH, who followed the criteria of study section to screen relevant articles. A consensus meet was performed to resolve any disagreement between two authors. The protocol of this NMA was registered at PROSPERO (registration number: CRD42021292060).

\subsection{Search Strategy}

The following keywords relating to individuals' conditions were used: "osteoarthritis" OR "gonarthritis" OR "degenerative cartilage." The following keywords relating to interventions were used: "extracorporeal shock wave therapy" OR "shockwave therapy." The detailed search formulas for each database are presented in online Table S1.

\subsection{Selection Criteria of Studies}

Articles which met the following criteria were included (Table 1): (1) the trial was conducted based on a design of two-arm or multiarm RCT, as well as a design of quasi RCT; (2) the trial recruited such patients who had a symptom or radiographic diagnosis of KOA; (3) treatment groups received ESWT alone or in combination with other noninjective treatments such as CPT and TCM; (4) the control group received a placebo ESWT, relatively low-dosage ESWT, or non-ESWT comparator intervention. Non-ESWT comparator interventions included CPT, TCM, and pain medication, all of which were classified as UC in this study; and (5) the study reported at least one of the primary or secondary outcome measures defined in Section 2.4.

Table 1. Criteria of study selection.

\begin{tabular}{ll}
\hline Trial design & Randomized controlled trial; quasirandomized controlled trial \\
Participant & Symptomatic or radiographic diagnosis of knee osteoarthritis \\
Treatment group & Received ESWT alone, ESWT plus CPT, or ESWT plus TCM \\
Control group & $\begin{array}{l}\text { Received a placebo ESWT, relatively low-dosage ESWT, or non-ESWT } \\
\text { intervention (i.e., CPT or TCM) }\end{array}$ \\
Outcome & Pain, global function, disease inflammation
\end{tabular}

CPT, conventional physical therapy; ESWT, extracorporeal shockwave therapy; TCM, traditional Chinese medicine.

Studies were eliminated if (1) the trial (or study arm) used intra-articular injections as a primary or comparator intervention; (2) the trial was conducted in vitro or in vivo by using an animal model; or (2) they were non-RCTs including case reports, case series, or prospectively designed trials without a comparison group.

\subsection{Outcome Measures}

The primary outcomes in this NMA included measures of pain and patient-perceived global function. Pain score was assessed using a quantifiable scale such as a visual analogue scale [44] or pain subscale derived from questionnaire-based instruments [45]. Global function was measured using self-administered instruments [45], which included the Western Ontario and McMaster Universities Arthritis Index (WOMAC) [46], Knee Injury and Osteoarthritis Outcome Score [47], Lequesne index [48], and Lysholm Knee Scoring Scale [49]. When the WOMAC total score was unavailable, the total sum of the scores derived from its subscales (i.e., pain, stiffness, and physical difficulty) were calculated, and other assessment tools were employed to measure global function. If the trial reported two or more global function scores, the priority of selection for analyses was as follows: WOMAC, Knee Injury and Osteoarthritis Outcome Score, Lysholm Knee Scoring Scale, and Lequesne index $[50,51]$.

The secondary outcome was the disease inflammation of KOA. Disease inflammation was measured using the intra-articular level of the inflammatory factors as follows: (1) proinflammatory cytokines such as interleukin 1 beta, tumor necrosis factor $\alpha$, and 
interleukin 6, which are involved in the pathogenesis of KOA [52]; (2) nitric oxide, which is associated with the disease progression of KOA [53,54]; and (3) synovial fluid adipokines such as chemerin, which are associated with the disease severity of KOA $[55,56]$.

\subsection{Data Extraction and Synthesis}

The following data were extracted from each included trial and presented in an evidence table (Table 2): (1) characteristics of study design and samples including the study arm, age, body mass index (BMI), sex distribution, and geographic area of the study population; (2) characteristics of disease onset including affected side, disease severity (Kellgren and Lawrence grade), and disease duration; (3) measured time points; and (4) main outcomes. If the trial had multiple treatment or control groups, only the study arms not related to intra-articular injections were selected and extracted for analyses. One of the team members, CDL, recorded the relevant data derived from the included RCTs, and the second team member, HCC, reviewed and confirmed the extracted data. If there was any discrepancy between the two team members, it was discussed and resolved through a consensus meeting. If the disagreements were not resolved, a third team member, CLL, was consulted for further judgement.

If the RCT separately reported treatment effects on bilateral legs, those of bilateral legs were combined to enable a single comparison, and such a procedure is recommended in the Cochrane Handbook for Systematic Reviews of Interventions [57]. The energy level of the ESWT was defined based on the EFD administered in each included trial and was classified as low $\left(<0.08 \mathrm{~mJ} / \mathrm{mm}^{2}\right)$, medium $\left(\geq 0.08 \mathrm{~mJ} / \mathrm{mm}^{2},<0.25 \mathrm{~mJ} / \mathrm{mm}^{2}\right)$, or high $\left(\geq 0.25 \mathrm{~mJ} / \mathrm{mm}^{2}\right)$ [58-61]. If the trial had multiple study arms with different EFDs, results of the same energy level were combined into a single treatment effect [57]. The duration of follow-up was assessed and defined as immediate ( $<1$ month), short ( $\geq 1$ and $<3$ months), medium ( $\geq 3$ and $<6$ months), or long term ( $\geq 6$ months) for subgroup analysis; when multiple time points were reported within the same timeframe, the analyzed results constituted those with the longest follow-up period for each of the included studies. For example, if the measured time points for pain score were 12 and 16 weeks in one trial, only the data from the 16-week follow-up were selected as medium-term results.

The presentation of the all-cause withdrawal rate was expressed to assess tolerance to the ESWT regimen. We also examined adverse events when reported; however, they were not specified a priori.

\subsection{Methodological Quality and Risks of Bias of Included Trials}

In this NMA, the PEDro quality score was used to rank the methodological quality and risk of bias. Two of the team members, $\mathrm{CDL}$ and $\mathrm{SWH}$, independently assessed methodological quality of each included RCT. The PEDro scale comprises 10 ranking items which correspond to selection bias (items 1-3: random allocation, concealed allocation, similarity at baseline); performance bias (items 4-5: subject blinding, therapist blinding); detection bias (items 6 and 10: assessor blinding, point and variability measures for at least one key outcome); attrition bias (items 7-8: $>85 \%$ follow-up for at least one key outcome, intentionto-treat analysis); and reporting bias (item 9: between-group statistical comparison for at least one key outcome). Validity of the PEDro scale has been identified [62]. The interrater reliability of ratings for the individual PEDro scale items varies from moderate to excellent (Kappa value: $0.53-0.94)$ for assessing the quality of RCTs [63]. In addition, an intraclass correlation coefficient for the PEDro total sum score has been identified as 0.91 [ $95 \%$ confidence interval (CI): 0.84-0.95] [64]. The methodological quality of the included RCTs was considered as low, medium, and high with a total PEDro score $\leq 3 / 10,4-6 / 10$, and $\geq 7 / 10$, respectively [65].

A funnel plot was used to identify potential publication bias by subjective visual assessment [66]. In addition, Egger's regression test was performed to identify any asymmetry in the funnel plot [67], which explored reporting bias. 
Table 2. Summary of study characteristics.

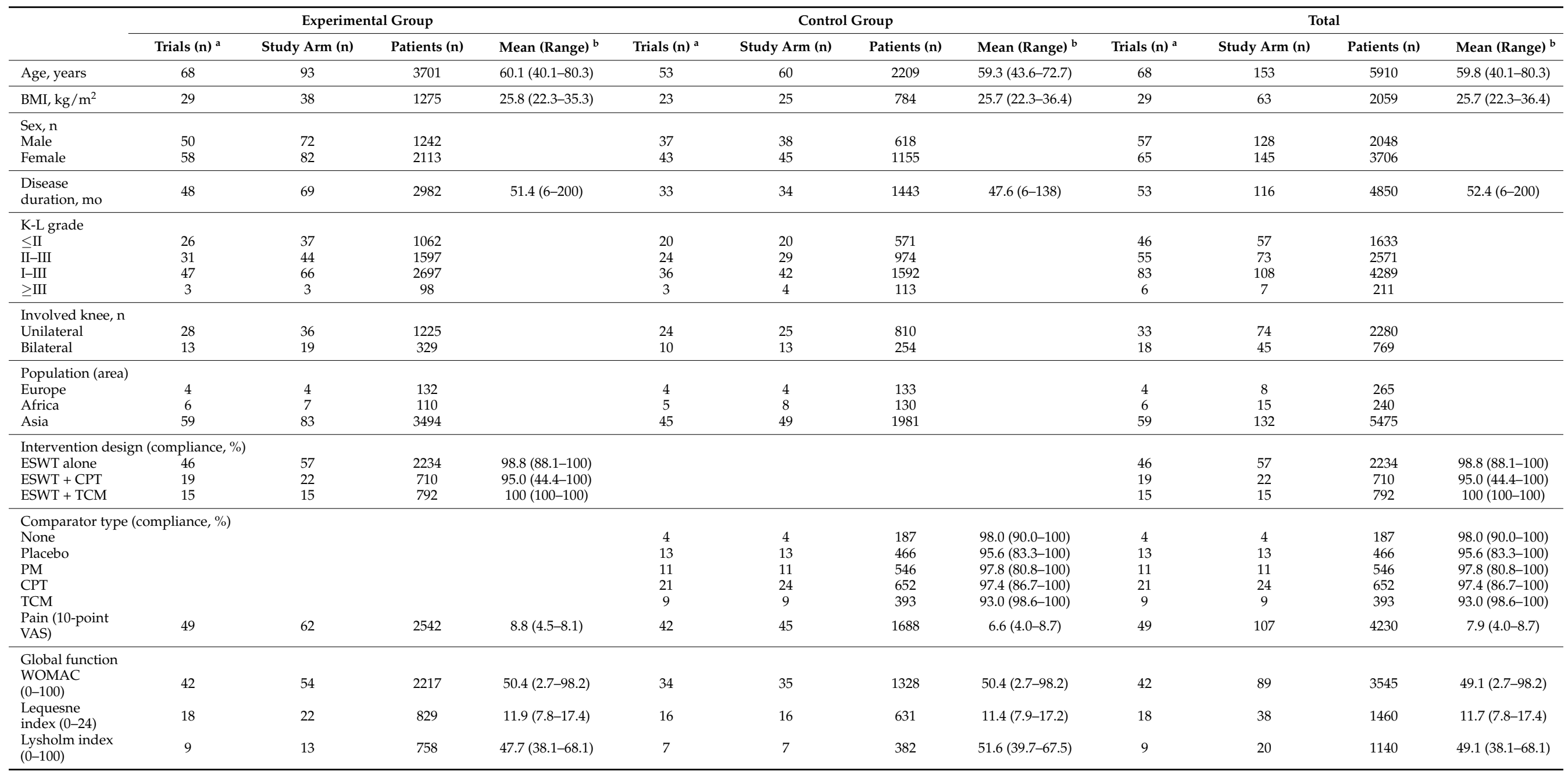


Table 2. Cont.

\begin{tabular}{|c|c|c|c|c|c|c|c|c|c|c|c|c|}
\hline & \multicolumn{4}{|c|}{ Experimental Group } & \multicolumn{4}{|c|}{ Control Group } & \multicolumn{4}{|c|}{ Total } \\
\hline & Trials $(\mathbf{n})^{a}$ & Study Arm (n) & Patients (n) & Mean (Range) ${ }^{b}$ & Trials (n) ${ }^{a}$ & Study Arm (n) & Patients (n) & Mean (Range) ${ }^{b}$ & Trials (n) a & Study Arm (n) & Patients (n) & Mean (Range) ${ }^{b}$ \\
\hline \multicolumn{13}{|c|}{ Disease inflammation } \\
\hline $\mathrm{IL}-1(\mathrm{pg} / \mathrm{mL})$ & 9 & 11 & 571 & $\begin{array}{c}75.6 \\
(17.3-220.9)\end{array}$ & 7 & 7 & 402 & $\begin{array}{c}49.6 \\
(16.9-113.4)\end{array}$ & 9 & 18 & 973 & $\begin{array}{c}65.5 \\
(16.9-220.9)\end{array}$ \\
\hline $\begin{array}{l}\text { Nitric oxide } \\
(\mu \mathrm{mol} / \mathrm{mL})\end{array}$ & 5 & 7 & 489 & $80.3(65.7-96.4)$ & 4 & 4 & 280 & $73.1(64.2-76.4)$ & 5 & 11 & 769 & $77.7(64.2-96.4)$ \\
\hline
\end{tabular}

a The number of trials that reported the indicated item. ${ }^{\mathrm{b}}$ All summations calculated based on the values reported in the included trials and that could be estimated. BMI, body mass index; CPT, conventional physical therapy; ESWT, extracorporeal shockwave therapy; IL-1, interleukin 1; K-L grade, Kellgren and Lawrence grade; PM, pain medicine; TCM, traditional Chinese medicine; TNF- $\alpha$, tumor necrosis factor $\alpha$; VAS, visual analogue scale. 


\subsection{Statistical Analysis}

The effect sizes on each outcome measure between any two study arms were computed in this NMA. The effect size was expressed as standard mean difference (SMD), which was calculated by dividing the between-group mean difference in the change score by the pooled standard deviation (SD). To partially correct betweenparticipant variability, all analyses were performed based on change scores (i.e., change from baseline) [57]. Where the change score in mean and SD was reported it was directly collected from the included RCT. If SD of change score was not reported for the outcome measure, it was estimated by the baseline and posttest measured SD in accordance with the Cochrane Handbook for Systematic Reviews of Interventions [57]. We followed Rosenthal's approach by assuming a within-participant correlation coefficient of 0.7 between the baseline and posttest measured data [68]. Where $p$ values or $95 \%$ CIs were reported instead of SDs, from which the SDs were calculated using the methods recommended by the Cochrane Handbook for Systematic Reviews of Interventions [57]. For trials reporting data as the median and interquartile range, the median was used to be representative of the mean value, and the interquartile range was divided by 1.35 to produce the SD [57].

The random-effects NMA were performed for all outcome measures using the frequentist methods. All analyses were performed using statistical software R (version 4.0.4, $\mathrm{R}$ Foundation for Statistical Computing, Vienna, Austria) [69,70]. Direct and indirect comparisons between different ESWT regimens were performed [71]. The Cochran's Q statistics or $I^{2}$ test was employed to assess heterogeneity along with $\tau^{2}$ values to estimate the variance across studies. In addition, we assessed inconsistency between direct and indirect comparisons using the node-splitting method [72,73]. Statistical significance was set at a two-way $p$ value less than 0.05 .

P score was used to rank the probabilities of effect estimation for each treatment [74]. Network forest plots which presented relative effects among treatment options using UC as reference were generated to identify the uncertainty in NMA [75].

Network metaregression analyses were performed to assess the confounding effects of potential moderators based on (1) participant characteristics including age, BMI, sex distribution (i.e., proportion of female participants in a sample), disease-onset duration, and area of the study population; (2) study methodology including level of methodological quality (i.e., PEDro score) and follow-up duration; and (3) intervention design including shockwave type, EFD level, treatment composition (i.e., monotherapy or combination treatment), and treatment duration.

Potential publication bias was assessed through the visual inspection of a funnel plot [76], and Egger's regression asymmetry test was performed to explore possible reporting bias [67].

\section{Results}

\subsection{Patient Demographics and Clinical Characteristics}

Figure 1 presents the selection process of eligible trials. A total of 800 articles were identified through an electronic and manual literature search, after which the duplicates were excluded. By reviewing the titles and abstracts, 238 studies were assessed for their eligibility, among which 114 were considered relevant for full-text assessment. Finally, the sample in this NMA comprised 70 articles on 69 RCTs published between 2000 and 2020. 


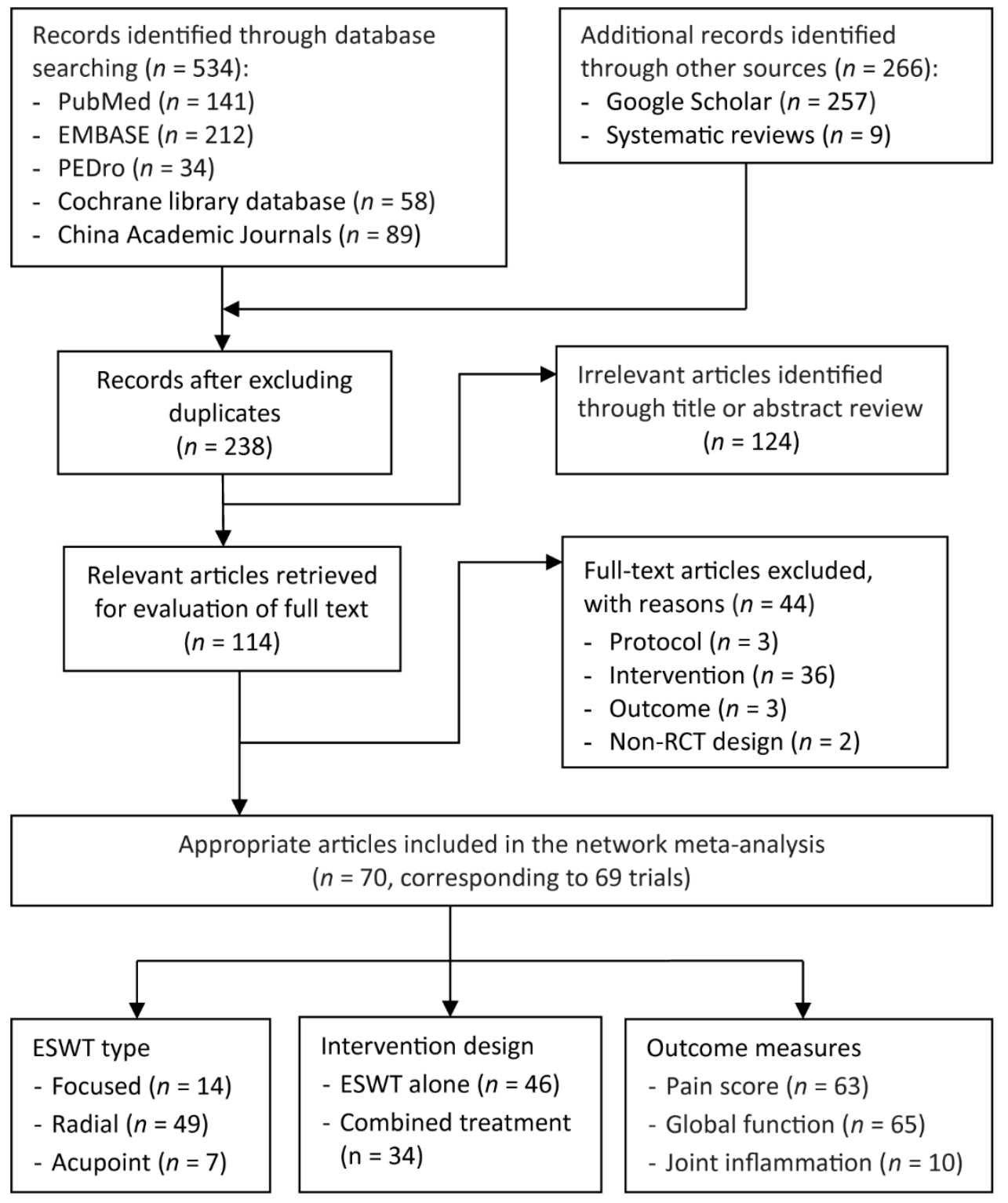

Figure 1. PRISMA flowchart of the study selection. ESWT, extracorporeal shockwave therapy; RCT, randomized control trial.

\subsection{Study Characteristics}

The study characteristics of and patient demographic data from the included RCTs were summarized in Table 2; the details of each trial are presented in Table S2. A total of 5980 participants who had received a diagnosis of symptomatic or radiographic KOA were recruited. Overall, the whole sample had a mean age of 59.8 (range: 40.1-80.3) years, mean BMI of 25.7 (range: $22.3-36.4$ ) kg/m² and mean disease duration of 52.4 (range: 6-200) months; the average proportion of female participants was 60.5\% (range: 10-94.4\%), which was estimated through the exclusion of eight sex-specific (female participants only) RCTs.

In this NMA, 54 of the included RCTs were two-arm studies, and the other 15 RCTs had a multiarm design, with a total of 155 study arms (ESWT, 94 arms). Among all participants, $2234(37.4 \%)$ received diet therapy alone, $1502(25.1 \%)$ received combination treatment, and 2244 (37.5\%) received UC (i.e., placebo or non-ESWT comparator). With respective to the follow-up duration, $67 \mathrm{RCTs}$ had an immediate or short-term follow-up duration of $<12$ weeks, 21 had a medium-term follow-up duration ranging from 12 to 19 weeks, and 10 had a long-term follow-up duration ranging from 6 to 14 months (Table S2). 


\subsection{ESWT Intervention Characteristics}

\subsubsection{ESWT Protocols}

The protocols for ESWT intervention employed in the included RCTs are summarized in Table S3. With respect to shockwave types, $14(20.2 \%)$ and $48(69.5 \%)$ out of the 69 included RCTs employed FoSW and RaSW, respectively, targeting tender points around the knee joint. Specifically, seven RCTs (10.3\%) applied acupoint therapy by using RaSW for patients with KOA. Regarding the shock energy level, 31 RCTs applied ESWT with an EFD of $\geq 0.25 \mathrm{~mJ} / \mathrm{mm}^{2}, 32$ used medium EFD, and nine employed low EFD (Table S3). In total, 65 RCTs applied an ESWT protocol consisting of three to 14 treatment sessions (one to three sessions weekly) within a treatment duration of 2 to 8 weeks, whereas 4 RCTs administered 15 to 30 treatment sessions within a treatment duration of 9 to 24 weeks. No local anesthesia was administered at the treatment site during application in all the included RCTs.

\subsubsection{Treatment Arms of ESWT}

In summary (Table 3), a total of 21 treatment arms of ESWT were identified and included in the NMA, which was based on three types of ESWT therapy (FoSW, RaSW, and acupoint ESWT), three levels of EFD (high, medium, and low), and three combination treatment regimens (ESWT alone, ESWT plus CPT, and ESWT plus TCM).

Table 3. Abbreviations for treatment arms.

\begin{tabular}{ll}
\hline Treatment Arm & Abbreviation \\
\hline Acupoint therapy using ESWT & Acupoint ESWT \\
Acupoint ESWT plus CPT & Acupoint ESWT + CPT \\
Acupoint ESWT plus TCM & Acupoint ESWT + TCM \\
\hline Radial shockwave & RaSW \\
High-energy radial shockwave & HI-RaSW \\
Medium-energy radial shockwave & MI-RaSW \\
Low-energy radial shockwave & LI-RaSW \\
High-energy radial shockwave plus CPT & HI-RaSW + CPT \\
Medium-energy radial shockwave plus CPT & MI-RaSW + CPT \\
Low-energy radial shockwave plus CPT & LI-RaSW + CPT \\
High-energy radial shockwave plus TCM & HI-RaSW + TCM \\
Medium-energy radial shockwave plus TCM & MI-RaSW + TCM \\
Low-energy radial shockwave plus TCM & LI-RaSW + TCM \\
\hline Focused shockwave & FoSW \\
High-energy focused shockwave & HI-FoSW \\
Medium-energy focused shockwave & MI-FoSW \\
Low-energy focused shockwave & LI-FoSW \\
High-energy focused shockwave plus CPT & HI-FoSW + CPT \\
Medium-energy focused shockwave plus CPT & MI-FoSW + CPT \\
Low-energy focused shockwave plus CPT & LI-FoSW + CPT \\
High-energy focused shockwave plus TCM & HI-FoSW + TCM \\
Medium-energy focused shockwave plus TCM & MI-FoSW + TCM \\
Low-energy focused shockwave plus TCM & LI-FoSW + TCM \\
\hline CPT, conventional physical therapy; ESWT, extracorporeal shockwave therapy; TCM, traditional Chinese medicine.
\end{tabular}

\subsection{Risk of Bias in Included Studies}

The detailed ratings of PEDro items of each included RCT are presented in Table S4. Overall, results of methodological quality assessment showed that 25 out of the $69(36.2 \%)$ included RCTs were classified as high methodological quality whereas the other 44 RCTs were considered as medium. The median PEDro score was estimated as 6 out of 10 (range: $5 / 10$ to $9 / 10)$ with an intraclass correlation coefficient of 0.98 (95\% CI: 0.96-0.98). In the view of the risk of bias across RCTs, all the 69 included RCTs employed random allocation, similarity at baseline, between-group comparisons, and point estimates and variability. In 
total, $16(64 \%), 2$ (8\%), and 23 (92\%) out of the 25 high-quality RCTs blinded the participants, therapists, and assessors, respectively, whereas only one medium-quality RCTs performed assessor blinding; none of the medium-quality RCTs blinded the participants or therapists. Moreover, 12 of the 25 (48\%) high-quality RCTs performed allocation concealment, as did one medium-quality RCT.

\subsection{Effectiveness of Treatment for Pain Reduction Assessed in NMA}

Figure 2 shows the network of eligible comparisons between any two of the treatment options for each outcome measure. Figures 3 and 4 present the relative effects of ESWT treatment on primary outcomes during an overall follow-up duration and at each time frame, and Figures S1 and S2 present the details of each comparison, respectively. In addition, Supplementary Tables S5-S7 demonstrate the league tables which provide results of pairwise meta-analysis and NMA.

(A) Pain

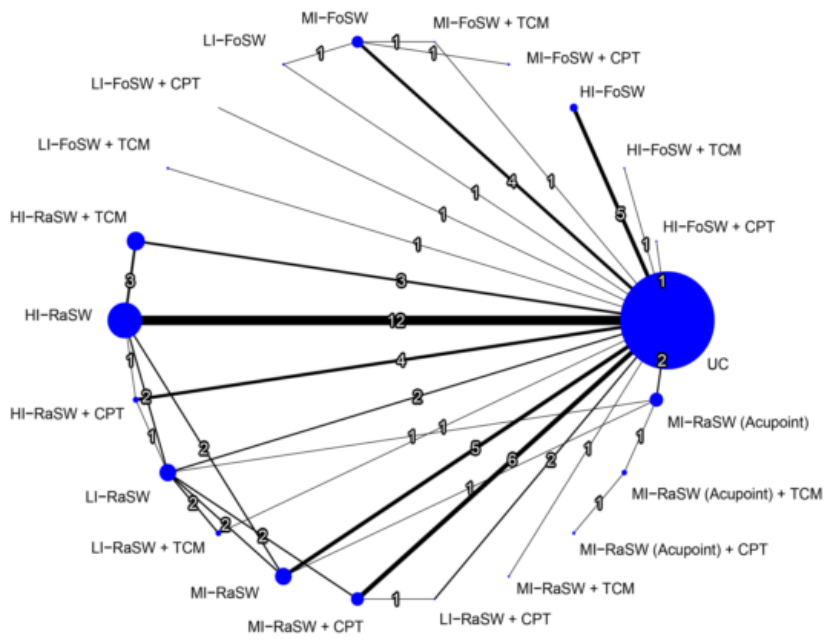

(B) Global function

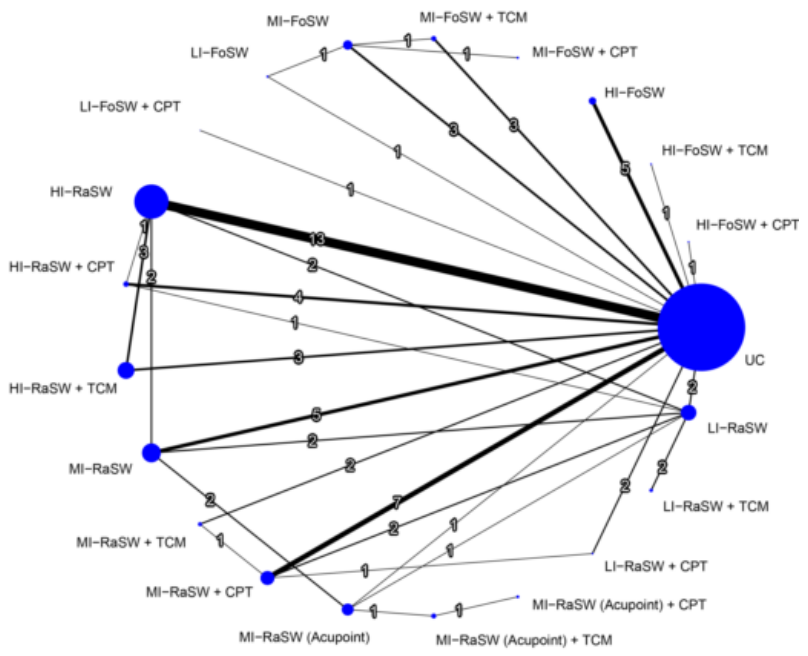

(C) Disease inflammation

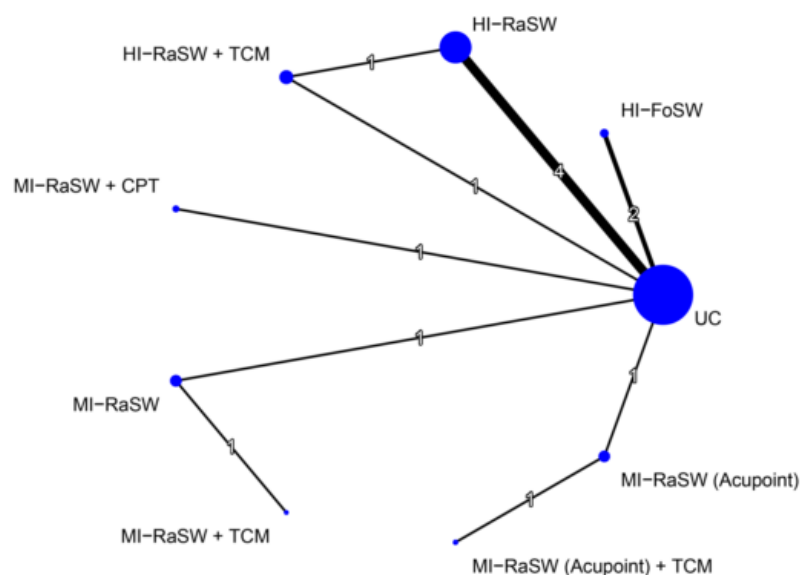

Figure 2. Network plot of the direct comparisons of different treatments for (A) pain, (B) global function, and (C) disease inflammation. The lines between nodes indicate direct comparisons in various studies. The size of each node is proportional to the number of the participants. The thickness of each line is proportional to the number of studies denoted on the line. FoSW, focused shockwave; RaSW, radial shockwave; HI, high energy; MI, medium energy; LI, low energy; CPT, conventional physical therapy; TCM, traditional Chinese medicine; UC, usual care. 


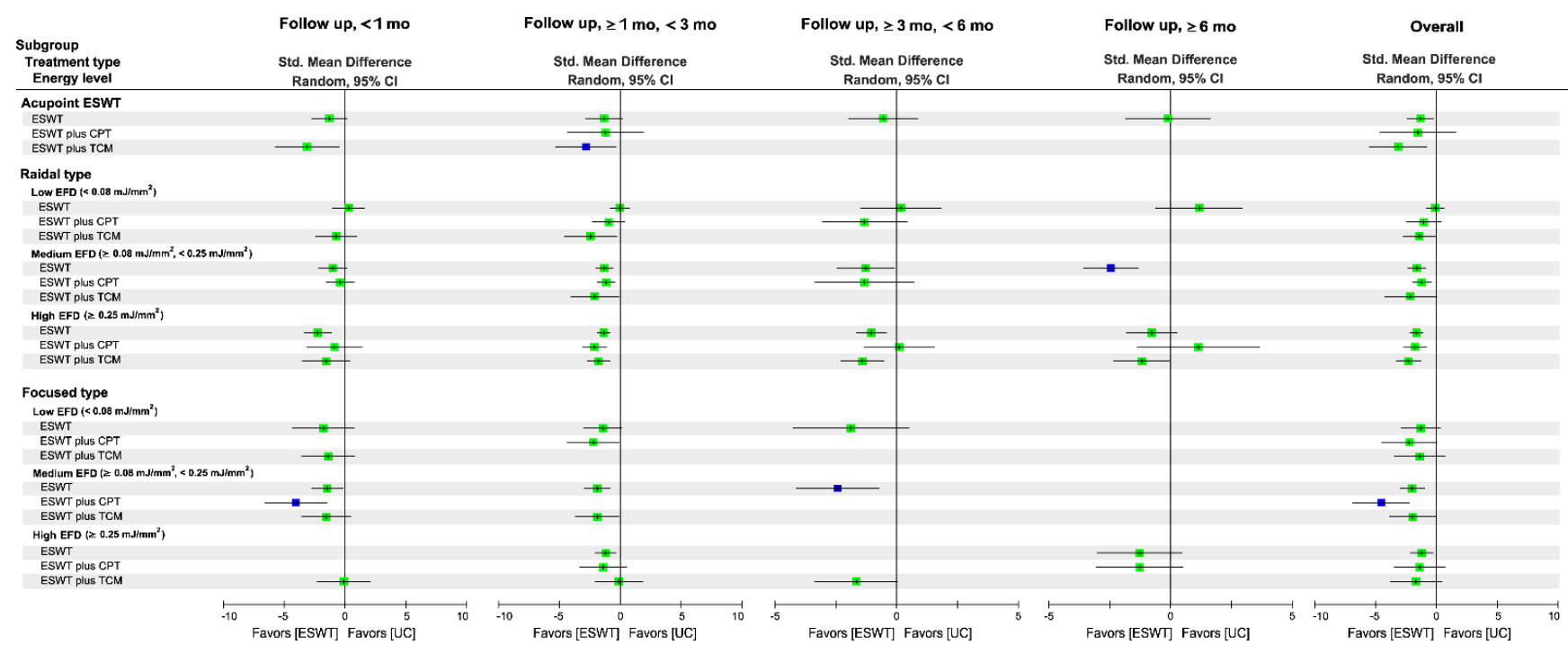

Figure 3. Relative effects among treatment regimens of ESWT on pain reduction at each timeframe and during an overall follow-up duration. The square point in each timeframe and for the overall duration presents the network combined effect (SMD) on changes in pain score relative to UC, and the horizontal line denotes a corresponded $95 \%$ CI. The highest rank of probability among all treatments in the same timeframe is denoted by a blue point. $95 \% \mathrm{CI}=95 \%$ confidence interval; mo, month; ESWT, extracorporeal shockwave therapy; EFD, energy flux density; CPT, conventional physical therapy; SMD, standard mean difference; TCM, traditional Chinese medicine; UC, usual care.

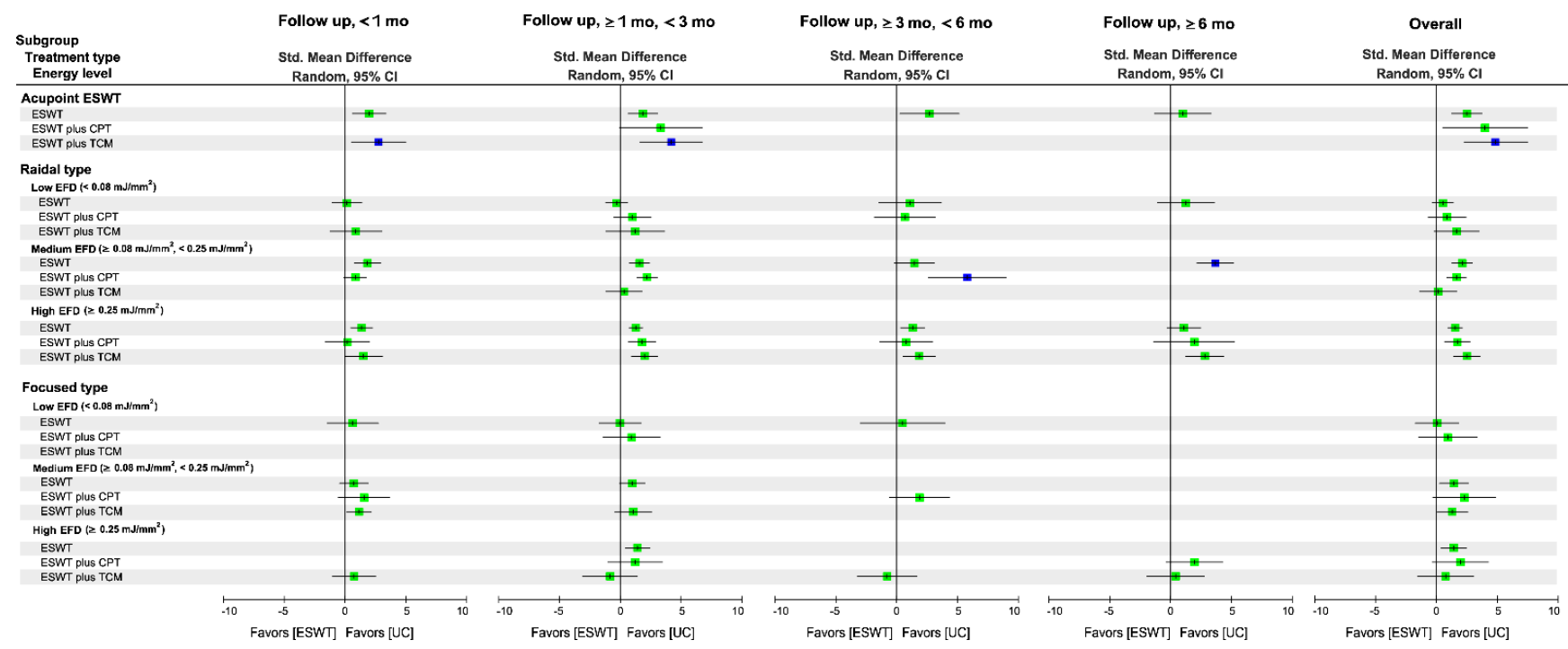

Figure 4. Relative effects among treatment regimens of ESWT on pain reduction at each timeframe and during an overall follow-up duration. The square point in each timeframe and for the overall duration presents the network combined effect (SMD) on changes in pain score relative to UC, and the horizontal line denotes a corresponded 95\% CI. The highest rank of probability among all treatments in the same timeframe is denoted by a blue point. $95 \% \mathrm{CI}=95 \%$ confidence interval; mo, month; ESWT, extracorporeal shockwave therapy; EFD, energy flux density; CPT, conventional physical therapy; SMD, standard mean difference; TCM, traditional Chinese medicine; UC, usual care.

\subsubsection{Pairwise Meta-Analysis}

Direct comparisons of pairwise meta-analyses (Table S5) indicated that FoSW applied with medium (SMD $=-1.98 ; 95 \% \mathrm{CI}:-3.03,-0.92)$ and high (SMD $=-1.21 ; 95 \% \mathrm{CI}:-2.15,-0.26)$ EFD were more efficacious than UC for pain reduction; in addition, medium-EFD (SMD = -1.64; 
95\% CI: $-2.60,-0.69)$ and high-EFD (SMD $=-1.68 ; 95 \%$ CI: $-2.29,-1.07)$ RaSW induced greater pain-related changes compared with UC and the combination treatments [medium-EFD RaSW plus CPT (SMD = -1.01; 95\% CI: $-1.88,-0.15)$; high-EFD RaSW plus either CPT (SMD $=-2.20 ; 95 \%$ CI: $-3.30,-1.09)$ or TCM (SMD $=-1.28 ; 95 \%$ CI: $-2.47,-0.09)]$.

In addition, the combination treatments yielded greater reductions in pain compared with ESWT alone; such results were observed in medium-EFD FoSW plus CPT (SMD $=-2.56$ 95\% CI: $-4.67,-0.45)$ and high-EFD RaSW plus TCM (SMD = -1.71; 95\% CI: $-2.91,-0.51)$ in comparison to medium-EFD FoSW and high-EFD RaSW alone, respectively.

\subsubsection{Global Effects in NMA}

The NMA for pain score was based on 62 RCTs (55 two-arm RCTs; 7 three-arm RCTs) with 22 treatments, 32 designs, and 76 pairwise comparisons. The NMA results demonstrated that, in comparison with UC, FoSW with medium (SMD = -1.95; $95 \% \mathrm{CI}:-2.95,-0.95)$ and high (SMD $=-1.21 ; 95 \% \mathrm{CI}:-2.15,-0.26)$ EFD induced greater changes in pain score-as did the RaSW with medium (SMD $=-1.61 ; 95 \%$ $\mathrm{CI}:-2.36,-0.86)$ and high $(\mathrm{SMD}=-1.64 ; 95 \% \mathrm{CI}:-2.19,-1.10)$ EFD-during the overall follow-up duration; additionally, acupoint ESWT using medium-EFD RaSW $(\mathrm{SMD}=-1.64 ; 95 \% \mathrm{CI}:-2.19,-1.10)$ resulted in greater reductions in pain score compared with UC (Figures 3 and S1). The combined effects of medium-EFD FoSW plus CPT $(\mathrm{SMD}=-4.51)$, high-EFD RaSW plus either CPT (SMD = -1.74) or TCM $(\mathrm{SMD}=-2.29)$, and acupoint ESWT plus TCM $(\mathrm{SMD}=-3.13)$ on pain reduction relative to UC were stronger than the effect of the solely FoSW, RaSW, and acupoint ESWT, respectively (Figures 3 and $\mathrm{S} 1$ ).

During the overall follow-up duration, medium-EFD FoSW plus CPT was ranked the most effective $(P$ score $=0.97$ ) among all treatment arms for pain reduction-followed by acupoint ESWT plus TCM (P score $=0.84)$, high-EFD RaSW plus TCM (P score $=0.74)$, and low-EFD FoSW plus CPT (P score $=0.66$; Figure S1). A significant global heterogeneity was observed $\left(\tau^{2}=1.07, I^{2}=94.2 \%, p<0.0001\right)$. According to the results of node-splitting analyses (Figure S3), there were no inconsistencies between the direct and indirect evidence for each comparison.

\subsubsection{Subgroup Analysis of Follow-Up Duration}

The combination treatment of medium-EFD FoSW plus CPT (SMD = -4.02; P score = 0.95) and medium-EFD FoSW alone $(\mathrm{SMD}=-2.43$; $\mathrm{P}$ score $=0.88$ ) were ranked as optimal treatments for pain reduction at the immediate and medium-term follow-up duration, respectively, whereas acupoint ESWT (i.e., medium-EFD RaSW) plus TCM (SMD = -2.83; P score $=0.83)$ and medium-EFD RaSW $(S M D=-2.40 ;$ P score $=0.96)$ were ranked the highest among all treatments over the short-term and long-term follow-up durations, respectively (Figures 3 and S1).

In summary, we determined that the combination treatments were the optimal ESWT protocols at immediate and short-term follow-up, whereas medium-EFD ESWT exhibited a higher ranked treatment effect on pain reduction at each follow-up timeframe, irrespective of the type of ESWT and its combination non-ESWT treatments.

\subsection{Effectiveness of Treatment for Global Function \\ 3.6.1. Pairwise Meta-Analysis}

Direct comparisons of pairwise meta-analyses (Table S6) indicated that FoSW applied with medium (SMD = 1.56, 95\% CI: 0.23-2.88) and high (SMD = 1.41, 95\% CI: 0.38-2.45) EFD were more efficacious than UC for function recovery, as were medium-EFD (SMD $=2.79,95 \% \mathrm{CI}$ : 1.72-3.86) and high-EFD (SMD = 1.65, 95\% CI: 1.01-2.29) RaSW; similar results were observed in the combined treatment medium-EFD RaSW plus CPT (SMD =1.61, 95\% CI: 0.73-2.49), as well as high-EFD RaSW combined with either CPT (SMD = 1.61, 95\% CI: 0.43-2.80) or TCM $(\mathrm{SMD}=1.88,95 \%$ CI: 0.57-3.19). 
In addition, acupoint ESWT plus TCM induced greater changes in function restoration than acupoint ESWT alone (SMD = 2.33, 95\% CI: 0.03-4.63); similar results were observed in the combination treatments of high-EFD RaSW plus CPT (SMD = 3.32, 95\% CI: 0.96-5.69) and high-EFD RaSW plus TCM (SMD = 1.44, 95\% CI: 0.12-2.75) compared with high-EFD RaSW alone (Table S6).

\subsubsection{Global Effects of NMA}

The NMA for global function was based on 63 RCTs (55 two-arm RCTs; eight three-arm RCTs) with 21 treatments, 32 designs, and 79 pairwise comparisons. The NMA results indicated that, during the overall follow-up duration, FoSW employed with medium $(\mathrm{SMD}=1.44 ; 95 \% \mathrm{CI}: 0.24-2.64)$ and high $(\mathrm{SMD}=1.41 ; 95 \% \mathrm{CI}: 0.38-2.45)$ EFD exerted significant effects on function regain relative to $U C$, as did the medium-EFD (SMD $=2.11$; 95\% CI: 1.28-2.93) and high-EFD (SMD = 1.54; 95\% CI: 0.96-2.12) RaSW (Figures 4 and S2); additionally, acupoint ESWT with medium-EFD RaSW led to a greater increase in function scores compared with UC (SMD $=2.46$; 95\% CI: 1.21-3.71). The combined effects of mediumEFD FoSW plus CPT (SMD = 2.29), high-EFD FoSW plus CPT (SMD = 1.97), high-EFD RaSW plus TCM (SMD = 2.50), and acupoint ESWT plus TCM $(S M D=4.97)$ on function restoration relative to UC were stronger than the effect of FoSW alone, RaSW alone, and acupoint ESWT alone, respectively (Figures 4 and S2).

Among all treatment arms for global function, acupoint ESWT plus TCM was ranked the most effective $(\mathrm{P}$ score $=0.97)$ followed by acupoint ESWT plus CPT $(\mathrm{SMD}=3.95$; P score $=0.87$ ), high-EFD RaSW plus TCM (P score $=0.78)$, and acupoint ESWT alone (SMD = 2.46, P score $=0.77$; Figures 4 and S2). A significant global heterogeneity was observed $\left(\tau^{2}=1.30\right.$, $I^{2}=95 \%, p<0.0001$ ). According to the results of node-splitting analyses (Figure S4), there were no inconsistencies between the direct and indirect evidence for each comparison.

\subsubsection{Subgroup Analysis of Follow-Up Duration}

Acupoint ESWT in combination with TCM was ranked the highest in terms of immediate $(\mathrm{SMD}=2.78 ; \mathrm{P}$ score $=0.90)$ and short-term $(\mathrm{SMD}=4.17 ; \mathrm{P}$ score $=0.96)$ treatment efficacy, respectively, for function restoration among all treatments (Figures 4 and S2). Additionally, medium-EFD RaSW plus CPT resulted in the highest medium-term treatment efficacy for global function (SMD $=5.75,95 \% \mathrm{CI}$ : 2.57-8.92; P score $=0.99$ ), as did mediumEFD RaSW alone in long-term follow-up (SMD = 3.70, 95\% CI: 2.18-5.21; P score = 0.93).

\subsection{Effectiveness of Treatment for Disease Inflammation}

\subsubsection{Pairwise Meta-Analysis}

Direct comparisons of the pairwise meta-analyses (Table S7) indicated that FoSW applying high EFD (SMD $=-5.01 ; 95 \%$ CI: $-6.98,-3.03)$ was more efficacious than UC for reducing disease inflammation.

\subsubsection{Global Effects of NMA}

The NMA for disease inflammation was based on 10 RCTs with two study arms and one RCT with a three-arm design. The NMA results demonstrated that, during the overall follow-up duration, high-EFD FoSW (SMD $=-5.01 ; 95 \%$ CI: $-6.98,-3.03$ ) exerted significant effects in terms of inflammation reduction relative to UC (Figure 5). High-EFD FoSW was ranked the most effective $(P$ score $=0.98$ ) among all treatment arms for disease inflammation followed by acupoint ESWT plus TCM $(\mathrm{SMD}=-2.21 ; \mathrm{P}$ score $=0.68)$ and medium-EFD RaSW plus TCM (SMD = -1.39; P score =0.53; Figure 5). A significant global heterogeneity was observed $\left(\tau^{2}=1.59, I^{2}=97.2 \%, p<0.0001\right)$. According to the results of node-splitting analyses (Figure S5), there were no inconsistencies between the direct and indirect evidence for each comparison. 
Treatment

Acupoint ESWT

MI-RaSW plus CPT

MI-RaSW

HI-RaSW plus TCM

HI-RaSW

Ml-RaSW plus TCM

Acupoint ESWT plus TCM

$\mathrm{HI}-\mathrm{FoSW}$
Direct

Comparisons

1
1
1
1
4
0
0
2

Random Effects Model

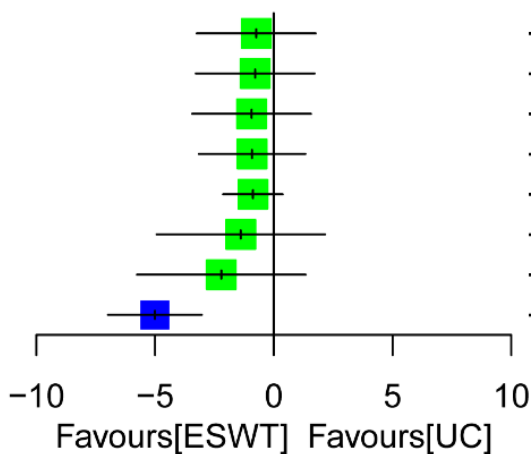

\section{SMD $\quad 95 \% \mathrm{Cl} \quad$ P-score}

$-0.74[-3.24 ; 1.76] \quad 0.39$

$-0.78[-3.28 ; 1.72] \quad 0.41$

$\begin{array}{lll}-0.94[-3.44 ; 1.56] & 0.44\end{array}$

$-0.92[-3.16 ; 1.33] \quad 0.44$

$-0.88[-2.13 ; 0.37] \quad 0.45$

$-1.39[-4.93 ; 2.15] \quad 0.53$

$-2.21[-5.76 ; 1.34] \quad 0.68$

$-5.01[-6.98 ;-3.03] \quad 0.98$

Figure 5. Forest plot summarizing the effects of treatment regimens of ESWT on disease inflammation for the overall follow-up duration. The blue point denotes the highest rank of probability, indicating that the treatment approach is the optimal intervention among all treatments. $95 \% \mathrm{CI}=95 \%$ confidence interval; CPT, conventional physical therapy; ESWT, extracorporeal shockwave therapy; FoSW, focused shockwave; HI, high energy; MI, medium energy; RaSW, radial shockwave; TCM, traditional Chinese medicine; UC, usual care.

\subsection{Network Metaregression Results for Moderators of Treatment Efficacy}

The NMA results are presented in supplementary Table S8. We observed that area of population $(\beta=0.87 ; p<0.05)$ and PEDro score $(\beta=-0.88 ; p<0.05)$ influenced ESWT efficacy for pain reduction. No moderator influenced treatment efficacy in terms of global function and disease inflammation.

\subsection{Compliance and Side Effects}

The rates of compliance with the ESWT interventions were $98 \%, 95 \%$, and $100 \%$ among the included RCTs that reported adherence to ESWT alone, ESWT plus CPT, and ESWT plus TCM, respectively, regardless of shockwave type or EFD level (Table 2).

No serious adverse events or severe complications were observed after ESWT alone or its combination treatments in all of the included RCTs. In total, 11 of the $69(15.9 \%)$ included RCTs reported side effects related to ESWT interventions, the most common of which related to treatment-induced knee pain, swelling, transient subcutaneous congestion, and short-term skin irritation (Table S9).

\subsection{Publication Bias}

The risk of publication bias across the included RCTs was considered low, since the distribution of the main outcomes in funnel plots did not show asymmetries (Figure S6). Egger's test results for pain outcome ( $p<0.0001$; Figure S6A) and global function $(p=0.0007$; Figure S6B) indicated significant reporting biases among the RCTs included in the NMA, whereas those for disease inflammation did not indicate any obvious reporting bias among said RCTs ( $p=0.597$; Figure S6C).

\section{Discussion}

The primary goal of this study was to identify the relative efficacy of different ESWT protocols and combination treatments for pain, global function, and disease inflammation (i.e., joint inflammation) outcomes in individuals with KOA. The NMA results demonstrated that (1) medium-EFD and high-EFD ESWT alone had overall significant effects on pain reduction, global function restoration, and disease inflammation reduction relative to UC, irrespective of the specific shockwave type or follow-up duration; (2) combination treatments (i.e., ESWT plus either CPT or TCM) exhibited additional treatment efficacy in terms of pain reduction and global function compared with ESWT alone; (3) in comparison with UC, FoSW achieved greater treatment efficacy in regard to pain reduction 
than RaSW, particularly medium-EFD ESWT, regardless of the specific intervention regime (i.e., ESWT alone or combination treatments); (4) based on the cumulative ranking results, medium-EFD FoSW plus CPT, acupoint ESWT plus TCM, and high-EFD FoSW alone were the optimal treatment strategies for reducing pain, restoring global function, and reducing disease inflammation, respectively.

This NMA is clinically useful in the context of the vast number of available treatment strategies and compositions for middle-aged or older adults with mild to moderate KOA. The most efficacious treatment option is not able to be determined by conservative pairwise meta-analysis, especially coupling the included trials with multiarm trials [77]. By contrast, without double counting of the participants among the included RCTs, the NMA methods provide consistent estimates of the relative effects among head-to-head treatment arms along with direct and indirect comparisons [78]. Additionally, treatment efficacy ranking of all of the identified ESWT protocols which were comprised of different shockwave types (i.e., FoSW alone or RaSW alone) and EFD levels is critical for its ability to elucidate the optimal approach among different treatment options for patients with KOA. Even with the lack of head-to-head RCTs, this NMA provides clinicians with the evidence regarding the comparative effects among different monotherapies and combination treatments of ESWT for patients with KOA, especially those who have contraindicated conditions for intra-articular injections or invasive treatments.

The results of this NMA revealed that, rather than high-EFD ESWT, the medium-EFD ESWT exhibited the strongest treatment effect on pain and function outcomes, irrespective of shockwave type. Our findings are consistent with those of another study indicating that ESWT applied at a medium dosage (EFD of $0.08-0.25 \mathrm{~mJ} / \mathrm{mm}^{2}$ ) produced greater effects in terms of pain as measured using WOMAC scores [41]. However, the metaregression results in this NMA indicated that a higher EFD predicts greater treatment effects on pain reduction and function restoration, despite statistical nonsignificance; accordingly, a highEFD $\left(\geq 2.5 \mathrm{~mJ} / \mathrm{mm}^{2}\right)$ ESWT is expected to yield greater treatment effects than medium-EFD ESWT. This inconsistency may be explained through the results indicating that a higher EFD is associated with higher ESWT treatment effects, which reach a peak at an EFD exceeding 0.32 (95\% CI: $0.22-0.42) \mathrm{mJ} / \mathrm{mm}^{2}$ for pain and 0.25 (95\% CI: $\left.0.21-0.29\right) \mathrm{mJ} / \mathrm{mm}^{2}$ for WOMAC scores [42]. Taken together, an ESWT employed at a medium-EFD dosage of 0.8 to $0.25 \mathrm{~mJ} / \mathrm{mm}^{2}$ may help achieve the optimal treatment outcome, particularly in regard to pain reduction and functional improvement.

Relative effects of FoSW and RaSW on pain reduction have been investigated and compared for musculoskeletal disorders [58,79-82] as well as KOA [41,42]. However, the results with respect to the superior treatment efficacy between the two shockwave types remain inconclusive. In this NMA, FoSW ranked the highest for pain reduction at immediate and medium-term follow-up, but RaSW had the highest ranking at short-term and long-term follow-up, particularly for the medium EFD treatment for KOA. Our findings are in agreement with the results of other studies comparing the treatment effectiveness of FoSW and RaSW subgroups in pain reduction for common soft tissue disorders of the knee [16]. We further verified that, when pooling the comparisons for all follow-up timeframes, medium-EFD FoSW plus CPT was ranked the most optimal treatment for pain reduction during the overall follow-up duration. Our finding indicates that the energy level may be the determinant of ESWT efficacy rather than the shockwave type for pain reduction.

In this study, the results of direct pairwise meta-analysis and NMA demonstrated that combination ESWT treatments incorporating CPT or TCM typically yielded greater effects for pain reduction and function restoration compared with ESWT alone, regardless of shockwave type or energy dosage. In addition, the combination treatments FoSW plus CPT and acupoint RaSW plus TCM were ranked the most effective treatment for pain and function outcomes, respectively. Our findings indicate that the combination treatment regimen of ESWT may be superior to ESWT monotherapy, which corroborates other subgroup analysis study results revealing a significant difference between adjunct 
treatment $(\mathrm{SMD}=-5.85)$ and monotherapy $(\mathrm{SMD}=-2.35)$ subgroups in terms of the WOMAC scores of patients with KOA [42].

Inflammatory factors are associated with KOA disease progression. Multiple studies have identified the effects of ESWT on the reduction of inflammation in animal models [83,84] and human osteoarthritic chondrocytes $[85,86]$. Analysis results in the present NMA revealed that high-EFD FoSW exerted significant effects on disease inflammation in terms of decrease in joint inflammation and was ranked the most optimal option among ESWT treatment arms. Our findings are consistent with those of other researchers and further indicate that ESWT, particularly FoSW, with an EFD greater or equal to $2.5 \mathrm{~mJ} / \mathrm{mm}^{2}$, exerts promising effects on joint inflammation for patients with KOA.

In this NMA, a series of metaregression models was established to identify potential moderators affecting relative efficacy among treatment arms. We observed no significant moderation effects in terms of participant characteristics, methodological level (i.e., PEDro score), follow-up duration, and intervention design for all primary and secondary outcomes, with the exception of area of population and PEDro score, both of which influenced ESWT efficacy for pain reduction. Two reasons may explain such findings. First, participants of an older age may have experienced minor pain reduction after ESWT, which may explain why relatively young (mean age $=49.6$ years) African patients experience greater pain reduction in response to ESWT compared with older (mean age $=61.5$ years) European patients. Second, as mentioned, medium-EFD ESWT had greater effects on pain outcome compared with high-EFD ESWT, which corresponds to the association between population area and treatment effects in relation to pain. Most of the RCTs that enrolled African patients employed medium-EFD ESWT, whereas those studying European patients applied an EFD of $0.4 \mathrm{~mJ} / \mathrm{mm}^{2}$; such findings further support that a medium EFD of 0.08 to $0.25 \mathrm{~mJ} / \mathrm{mm}^{2}$ is the optimal intervention dosage of ESWT rather than a high EFD of $\geq 2.5 \mathrm{~mJ} / \mathrm{mm}^{2}$.

The findings of this NMA must be interpreted considering the following limitations. First, because of the variation in the prescriptions of CPT or TCM (e.g., various exercise training programs, modality therapies, and Chinese pain medications) and ESWT application parameters (i.e., number of shocks, impulse frequency, and depth of energy), it was difficult to draw a definite conclusion regarding the effect of a specific ESWT protocol (such as dosage, number of impulses, and total number of treatment sessions) on pain reduction or function restoration. Second, all the non-ESWT comparators were pooled within a UC group. Because different non-ESWT comparators may have exerted influence in the pooled effects when all comparisons in the NMA were combined, the results must be interpreted with caution. Fourth, the estimates for treatment arms, including commination treatments of high-EFD and low-EFD FoSW, and acupoint ESWT plus CPT, were demonstrated with wide $95 \%$ CIs, which were subject to considerable uncertainty. Finally, for the treatment outcome of disease inflammation, the inadequate statistical power derived from small number of treatment arms may hinder the detection of inconsistency, despite of that inconsistency was not detected in the current NMA.

\section{Conclusions}

This NMA determined the relative efficacy of different ESWT regimens (i.e., shockwave type and energy level) and combination treatments (i.e., ESWT plus CPT or TCM) in terms of pain, global function, and disease inflammation in individuals with $\mathrm{KOA}$; in addition, medium-EFD FoSW plus CPT was determined to be the optimal treatment strategy for pain reduction, whereas acupoint ESWT plus TCM and high-EFD FoSW alone were the optimal treatment options for function restoration and inflammation inhibition, respectively, regardless of the intervention type or follow-up duration. Based on the analyses results, we conclude that ESWT alone reduces joint inflammation, and a combination treatment incorporating ESWT with an adjunct treatment (such as CPT or TCM), especially medium EFD, exerts favorable effects on pain reduction and functional improvement in individuals with KOA. Moreover, the study results contribute to the knowledge of optimal ESWT intervention strategies, emphasizing the need for a combination treatment to manage 
pain and functional decline in individuals with KOA. The findings of this NMA provide evidence for clinicians regarding the optimal ESWT regimens to ensure successful treatment outcomes. Based on the limitations of this NMA, additional studies enrolling large number of participants are warranted to further identify specific intervention protocols.

Supplementary Materials: The following are available online at https: / / www.mdpi.com/article / 10.3390/biomedicines10020306/s1. Table S1. Database search strategies; Table S2. Characteristics of the included studies; Table S3. Summary of characteristics and application parameters of extracorporeal shockwave therapy; Table S4. Ratings of the PEDro classification scale; Table S5. Direct pairwise meta-analysis and network meta-analysis results for pain reduction; Table S6. Direct pairwise comparison and network meta-analysis results for global function; Table S7. Direct pairwise meta-analysis and network meta-analysis results for disease inflammation; Table S8. Associations of moderators with treatment efficiency for all outcome measures; Table S9. All-cause withdraw and adverse events for included trials; Figure S1. Effects of shockwave therapy and combined treatments on changes of pain score; Figure S2. Effects of shockwave therapy and combined treatments on changes of global function; Figure S3. Node splitting results for pain outcome; Figure S4. Node splitting results for global function; Figure S5. Node splitting results for disease inflammation; Figure S6. Funnel plots for (A) pain, (B) global function, and (C) disease inflammation.

Author Contributions: Conceptualization: C.-D.L., C.-L.L. and S.-W.H.; investigation and validation: C.-D.L., H.-C.C. and C.-L.L.; methodology: C.-D.L., H.-C.C. and T.-H.L.; data curation and formal analysis: C.-D.L. and H.-C.C.; writing-original draft preparation: C.-D.L. and S.-W.H.; writing-review and editing: Y.-Y.H., H.-C.C. and T.-H.L.; taking the primary responsibility for the final content of the paper: C.-D.L. and S.-W.H. All authors have read and agreed to the published version of the manuscript.

Funding: Taipei Medical University-Shuang Ho Hospital, Ministry of Health and Welfare, Taiwan (grant no. 110TMU-SHH-14).

Institutional Review Board Statement: Not applicable.

Informed Consent Statement: Not applicable.

Data Availability Statement: Refer to Supplementary Materials. Raw data available on request.

Conflicts of Interest: The authors declare no conflict of interest.

\section{References}

1. Di Nicola, V. Degenerative osteoarthritis a reversible chronic disease. Regen. Ther. 2020, 15, 149-160. [CrossRef] [PubMed]

2. Katz, J.N.; Arant, K.R.; Loeser, R.F. Diagnosis and Treatment of Hip and Knee Osteoarthritis: A Review. JAMA 2021, 325, 568-578. [CrossRef]

3. Madry, H.; Kon, E.; Condello, V.; Peretti, G.M.; Steinwachs, M.; Seil, R.; Berruto, M.; Engebretsen, L.; Filardo, G.; Angele, P. Early osteoarthritis of the knee. Knee Surg. Sports Traumatol. Arthrosc. 2016, 24, 1753-1762. [CrossRef] [PubMed]

4. Puntillo, F.; Giglio, M.; Paladini, A.; Perchiazzi, G.; Viswanath, O.; Urits, I.; Sabbà, C.; Varrassi, G.; Brienza, N. Pathophysiology of musculoskeletal pain: A narrative review. Ther. Adv. Musculoskelet. Dis. 2021, 13, 1759720X21995067. [CrossRef] [PubMed]

5. Hunter, D.J.; Bierma-Zeinstra, S. Osteoarthritis. Lancet 2019, 393, 1745-1759. [CrossRef]

6. Hawker, G.A. Osteoarthritis is a serious disease. Clin. Exp. Rheumatol. 2019, 37 (Suppl. 120), 3-6.

7. Zitko, P.; Bilbeny, N.; Balmaceda, C.; Abbott, T.; Carcamo, C.; Espinoza, M. Prevalence, burden of disease, and lost in health state utilities attributable to chronic musculoskeletal disorders and pain in Chile. BMC Public Health 2021, 21, 937. [CrossRef]

8. Kiadaliri, A.A.; Lamm, C.J.; De Verdier, M.G.; Engström, G.; Turkiewicz, A.; Lohmander, S.; Englund, M. Association of knee pain and different definitions of knee osteoarthritis with health-related quality of life: A population-based cohort study in southern Sweden. Health Qual. Life Outcomes 2016, 14, 121. [CrossRef]

9. Kim, I.J.; Kim, H.A.; Seo, Y.-I.; Jung, Y.O.; Song, Y.W.; Jeong, J.Y.; Kim, D.H. Prevalence of knee pain and its influence on quality of life and physical function in the Korean elderly population: A community based cross-sectional study. J. Korean Med. Sci. 2011, 26, 1140-1146. [CrossRef]

10. Usiskin, I.M.; Yang, H.Y.; Deshpande, B.R.; Collins, J.E.; Michl, G.L.; Smith, S.R.; Klara, K.M.; Selzer, F.; Katz, J.N.; Losina, E. Association between activity limitations and pain in patients scheduled for total knee arthroplasty. BMC Musculoskelet. Disord. 2016, 17, 378. [CrossRef]

11. Davison, M.J.; Ioannidis, G.; Maly, M.R.; Adachi, J.D.; Beattie, K.A. Intermittent and constant pain and physical function or performance in men and women with knee osteoarthritis: Data from the osteoarthritis initiative. Clin. Rheumatol. 2016, 35, 371-379. [CrossRef] [PubMed] 
12. Lamb, S.E.; Guralnik, J.M.; Buchner, D.M.; Ferrucci, L.M.; Hochberg, M.C.; Simonsick, E.M.; Fried, L.P. Factors that modify the association between knee pain and mobility limitation in older women: The Women's Health and Aging Study. Ann. Rheum. Dis. 2000, 59, 331-337. [CrossRef] [PubMed]

13. Whitchelo, T.; McClelland, J.A.; Webster, K.E. Factors associated with stair climbing ability in patients with knee osteoarthritis and knee arthroplasty: A systematic review. Disabil. Rehabil. 2014, 36, 1051-1060. [CrossRef] [PubMed]

14. Korakakis, V.; Whiteley, R.; Tzavara, A.; Malliaropoulos, N. The effectiveness of extracorporeal shockwave therapy in common lower limb conditions: A systematic review including quantification of patient-rated pain reduction. Br. J. Sports Med. 2018, 52, 387-407. [CrossRef] [PubMed]

15. Liao, C.D.; Tsauo, J.Y.; Chen, H.C.; Liou, T.H. Efficacy of Extracorporeal Shock Wave Therapy for Lower Extremity Tendinopathy: A Meta-analysis of Randomised Controlled Trials. Am. J. Phys. Med. Rehabil. 2018, 97, 605-619. [CrossRef] [PubMed]

16. Liao, C.D.; Xie, G.M.; Tsauo, J.Y.; Chen, H.C.; Liou, T.H. Efficacy of extracorporeal shock wave therapy for knee tendinopathies and other soft tissue disorders: A meta-analysis of randomized controlled trials. BMC Musculoskelet. Disord. 2018, 19, 278. [CrossRef]

17. Mani-Babu, S.; Morrissey, D.; Waugh, C.; Screen, H.; Barton, C. The effectiveness of extracorporeal shock wave therapy in lower limb tendinopathy: A systematic review. Am. J. Sports Med. 2015, 43, 752-761. [CrossRef]

18. Leal, C.; Ramon, S.; Furia, J.; Fernandez, A.; Romero, L.; Hernandez-Sierra, L. Current concepts of shockwave therapy in chronic patellar tendinopathy. Int. J. Surg. 2015, 24, 160-164. [CrossRef]

19. Langer, P.R. Two emerging technologies for achilles tendinopathy and plantar fasciopathy. Clin. Podiatr. Med. Surg. 2015, 32, 183-193. [CrossRef]

20. Smith, W.B.; Melton, W.; Davies, J. Midsubstance Tendinopathy, Percutaneous Techniques (Platelet-Rich Plasma, Extracorporeal Shock Wave Therapy, Prolotherapy, Radiofrequency Ablation). Clin. Podiatr. Med. Surg. 2017, 34, 161-174. [CrossRef]

21. Vetrano, M.; d'Alessandro, F.; Torrisi, M.R.; Ferretti, A.; Vulpiani, M.C.; Visco, V. Extracorporeal shock wave therapy promotes cell proliferation and collagen synthesis of primary cultured human tenocytes. Knee Surg. Sports Traumatol. Arthrosc. 2011, 19, 2159-2168. [CrossRef] [PubMed]

22. Leone, L.; Raffa, S.; Vetrano, M.; Ranieri, D.; Malisan, F.; Scrofani, C.; Vulpiani, M.C.; Ferretti, A.; Torrisi, M.R.; Visco, V. Extracorporeal Shock Wave Treatment (ESWT) enhances the in vitro-induced differentiation of human tendon-derived stem/progenitor cells (hTSPCs). Oncotarget 2016, 7, 6410-6423. [CrossRef] [PubMed]

23. de Girolamo, L.; Stanco, D.; Galliera, E.; Viganò, M.; Lovati, A.; Marazzi, M.G.; Romeo, P.; Sansone, V. Soft-focused extracorporeal shock waves increase the expression of tendon-specific markers and the release of anti-inflammatory cytokines in an adherent culture model of primary human tendon cells. Ultrasound Med. Biol. 2014, 40, 1204-1215. [CrossRef]

24. Wang, C.J.; Cheng, J.H.; Chou, W.Y.; Hsu, S.L.; Chen, J.H.; Huang, C.Y. Changes of articular cartilage and subchondral bone after extracorporeal shockwave therapy in osteoarthritis of the knee. Int. J. Med. Sci. 2017, 14, 213-223. [CrossRef]

25. Chu, C.H.; Yen, Y.S.; Chen, P.L.; Wen, C.Y. Repair of articular cartilage in rabbit osteochondral defects promoted by extracorporeal shock wave therapy. Shock Waves 2015, 25, 205-214. [CrossRef]

26. Simplicio, C.L.; Purita, J.; Murrell, W.; Santos, G.S.; Dos Santos, R.G.; Lana, J. Extracorporeal shock wave therapy mechanisms in musculoskeletal regenerative medicine. J. Clin. Orthop. Trauma 2020, 11, S309-S318. [CrossRef]

27. Cheing, G.L.; Chang, H. Extracorporeal shock wave therapy. J. Orthop. Sports Phys. Ther. 2003, 33, 337-343. [CrossRef]

28. Ogden, J.A.; Toth-Kischkat, A.; Schultheiss, R. Principles of shock wave therapy. Clin. Orthop. Relat. Res. 2001, 387, 8-17. [CrossRef]

29. van der Worp, H.; van den Akker-Scheek, I.; van Schie, H.; Zwerver, J. ESWT for tendinopathy: Technology and clinical implications. Knee Surg. Sports Traumatol. Arthrosc. 2013, 21, 1451-1458. [CrossRef]

30. Foldager, C.B.; Kearney, C.; Spector, M. Clinical application of extracorporeal shock wave therapy in orthopedics: Focused versus unfocused shock waves. Ultrasound Med. Biol. 2012, 38, 1673-1680. [CrossRef]

31. Schmitz, C.; Császár, N.B.M.; Milz, S.; Schieker, M.; Maffulli, N.; Rompe, J.-D.; Furia, J.P. Efficacy and safety of extracorporeal shock wave therapy for orthopedic conditions: A systematic review on studies listed in the PEDro database. Br. Med. Bull. 2015, 116, 115-138. [CrossRef]

32. Speed, C. A systematic review of shockwave therapies in soft tissue conditions: Focusing on the evidence. Br. J. Sports Med. 2014, 48, 1538-1542. [CrossRef]

33. Storheim, K.; Gjersing, L.; Bolstad, K.; Risberg, M.A. Extracorporeal shock wave therapy (ESWT) and radial extracorporeal shock wave therapy (rESWT) in chronic musculoskeletal pain. Tidsskr. Nor. Laegeforen. 2010, 130, 2360-2364. [CrossRef]

34. Rompe, J.D.; Furia, J.; Weil, L.; Maffulli, N. Shock wave therapy for chronic plantar fasciopathy. Br. Med. Bull. 2007, 81-82, 183-208. [CrossRef]

35. Speed, C.A. Extracorporeal shock-wave therapy in the management of chronic soft-tissue conditions. J. Bone Jt. Surg. Br. 2004, 86, 165-171. [CrossRef]

36. Notarnicola, A.; Maccagnano, G.; Tafuri, S.; Fiore, A.; Margiotta, C.; Pesce, V.; Moretti, B. Prognostic factors of extracorporeal shock wave therapy for tendinopathies. Musculoskelet. Surg. 2016, 100, 53-61. [CrossRef]

37. Wang, Y.C.; Huang, H.T.; Huang, P.J.; Liu, Z.M.; Shih, C.L. Efficacy and Safety of Extracorporeal Shockwave Therapy for Treatment of Knee Osteoarthritis: A Systematic Review and Meta-analysis. Pain Med. 2020, 21, 822-835. [CrossRef] 
38. Ma, H.; Zhang, W.; Shi, J.; Zhou, D.; Wang, J. The efficacy and safety of extracorporeal shockwave therapy in knee osteoarthritis: A systematic review and meta-analysis. Int. J. Surg. 2020, 75, 24-34. [CrossRef]

39. Hsieh, C.K.; Chang, C.J.; Liu, Z.W.; Tai, T.W. Extracorporeal shockwave therapy for the treatment of knee osteoarthritis: A meta-analysis. Int. Orthop. 2020, 44, 877-884. [CrossRef]

40. Chen, L.; Ye, L.; Liu, H.; Yang, P.; Yang, B. Extracorporeal Shock Wave Therapy for the Treatment of Osteoarthritis: A Systematic Review and Meta-Analysis. BioMed Res. Int. 2020, 2020, 1907821. [CrossRef]

41. Avendaño-Coy, J.; Comino-Suárez, N.; Grande-Muñoz, J.; Avendaño-López, C.; Gómez-Soriano, J. Extracorporeal shockwave therapy improves pain and function in subjects with knee osteoarthritis: A systematic review and meta-analysis of randomized clinical trials. Int. J. Surg. 2020, 82, 64-75. [CrossRef] [PubMed]

42. Liao, C.D.; Tsauo, J.Y.; Liou, T.H.; Chen, H.C.; Huang, S.W. Clinical efficacy of extracorporeal shockwave therapy for knee osteoarthritis: A systematic review and meta-regression of randomized controlled trials. Clin. Rehabil. 2019, 33, 1419-1430. [CrossRef] [PubMed]

43. Li, T.; Ma, J.; Zhao, T.; Gao, F.; Sun, W. Application and efficacy of extracorporeal shockwave treatment for knee osteoarthritis: A systematic review and meta-analysis. Exp. Ther. Med. 2019, 18, 2843-2850. [CrossRef] [PubMed]

44. Atisook, R.; Euasobhon, P.; Saengsanon, A.; Jensen, M.P. Validity and Utility of Four Pain Intensity Measures for Use in International Research. J. Pain Res. 2021, 14, 1129-1139. [CrossRef]

45. Collins, N.J.; Misra, D.; Felson, D.T.; Crossley, K.M.; Roos, E.M. Measures of knee function: International Knee Documentation Committee (IKDC) Subjective Knee Evaluation Form, Knee Injury and Osteoarthritis Outcome Score (KOOS), Knee Injury and Osteoarthritis Outcome Score Physical Function Short Form (KOOS-PS), Knee Outcome Survey Activities of Daily Living Scale (KOS-ADL), Lysholm Knee Scoring Scale, Oxford Knee Score (OKS), Western Ontario and McMaster Universities Osteoarthritis Index (WOMAC), Activity Rating Scale (ARS), and Tegner Activity Score (TAS). Arthritis Care Res. 2011, 63 (Suppl. 11), S208-S228

46. Bilbao, A.; Martín-Fernández, J.; García-Pérez, L.; Arenaza, J.C.; Ariza-Cardiel, G.; Ramallo-Fariña, Y.; Ansola, L. Mapping WOMAC Onto the EQ-5D-5L Utility Index in Patients With Hip or Knee Osteoarthritis. Value Health 2020, 23, 379-387. [CrossRef]

47. Roos, E.M.; Roos, H.P.; Lohmander, L.S.; Ekdahl, C.; Beynnon, B.D. Knee Injury and Osteoarthritis Outcome Score (KOOS)-development of a self-administered outcome measure. J. Orthop. Sports Phys. Ther. 1998, 28, 88-96. [CrossRef]

48. Lequesne, M.G.; Mery, C.; Samson, M.; Gerard, P. Indexes of severity for osteoarthritis of the hip and knee. Validation-value in comparison with other assessment tests. Scand. J. Rheumatol. Suppl. 1987, 65, 85-89. [CrossRef]

49. Wang, W.; Liu, L.; Chang, X.; Jia, Z.Y.; Zhao, J.Z.; Xu, W.D. Cross-cultural translation of the Lysholm knee score in Chinese and its validation in patients with anterior cruciate ligament injury. BMC Musculoskelet. Disord. 2016, 17, 436. [CrossRef]

50. Metsavaht, L.; Leporace, G.; de Mello Sposito, M.M.; Riberto, M.; Batista, L.A. What is the best questionnaire for monitoring the physical characteristics of patients with knee osteoarthritis in the Brazilian population? Rev. Bras. Ortop. 2011, 46, $256-261$. [CrossRef]

51. Wang, D.; Jones, M.H.; Khair, M.M.; Miniaci, A. Patient-reported outcome measures for the knee. J. Knee Surg. 2010, 23 , 137-151. [CrossRef]

52. Molnar, V.; Matišić, V.; Kodvanj, I.; Bjelica, R.; Jeleč, Ž.; Hudetz, D.; Rod, E.; Čukelj, F.; Vrdoljak, T.; Vidović, D. Cytokines and Chemokines Involved in Osteoarthritis Pathogenesis. Int. J. Mol. Sci. 2021, 22, 9208. [CrossRef]

53. Hancock, C.M.; Riegger-Krugh, C. Modulation of pain in osteoarthritis: The role of nitric oxide. Clin. J. Pain 2008, 24, 353-365. [CrossRef]

54. Vuolteenaho, K.; Moilanen, T.; Knowles, R.G.; Moilanen, E. The role of nitric oxide in osteoarthritis. Scand. J. Rheumatol. 2007, 36, 247-258. [CrossRef]

55. Calvet, J.; Orellana, C.; Gratacós, J.; Berenguer-Llergo, A.; Caixàs, A.; Chillarón, J.J.; Pedro-Botet, J.; García-Manrique, M.; Navarro, N.; Larrosa, M. Synovial fluid adipokines are associated with clinical severity in knee osteoarthritis: A cross-sectional study in female patients with joint effusion. Arthritis Res. Ther. 2016, 18, 207. [CrossRef]

56. Carrión, M.; Frommer, K.W.; Pérez-García, S.; Müller-Ladner, U.; Gomariz, R.P.; Neumann, E. The Adipokine Network in Rheumatic Joint Diseases. Int. J. Mol. Sci. 2019, 20, 4190. [CrossRef]

57. Higgins, J.P.T.; Li, T.; Deeks, J.J. Cochrane Handbook for Systematic Reviews of Interventions. In Cochrane Handbook for Systematic Reviews of Interventions; Higgins, J.P., Thomas, J., Chandler, J., Cumpston, M., Li, T., Page, M.J., Welch, V.A., Eds.; The Cochrane Collaboration: London, UK, 2021.

58. Chang, K.V.; Chen, S.Y.; Chen, W.S.; Tu, Y.K.; Chien, K.L. Comparative effectiveness of focused shock wave therapy of different intensity levels and radial shock wave therapy for treating plantar fasciitis: A systematic review and network meta-analysis. Arch. Phys. Med. Rehabil. 2012, 93, 1259-1268. [CrossRef]

59. Lei, H.; Liu, J.; Li, H.; Wang, L.; Xu, Y.; Tian, W.; Lin, G.; Xin, Z. Low-intensity shock wave therapy and its application to erectile dysfunction. World J. Mens. Health 2013, 31, 208-214. [CrossRef]

60. Bannuru, R.R.; Flavin, N.E.; Vaysbrot, E.; Harvey, W.; McAlindon, T. High-energy extracorporeal shock-wave therapy for treating chronic calcific tendinitis of the shoulder: A systematic review. Ann. Intern. Med. 2014, 160, 542-549. [CrossRef]

61. Liu, B.Y.; Li, H.Y.; Xi, L.C.; Huang, S.C.; Wei, M.Z.; Wang, H.R. Comparison of early clinical outcomes of extracorporeal shock wave therapy in different energy flux densities for moderate knee osteoarthritis. Orthop. J. China 2020, 28, 908-912. 
62. Wu, C.-H.; Chen, K.-T.; Hou, M.-T.; Chang, Y.-F.; Chang, C.-S.; Liu, P.-Y.; Wu, S.-J.; Chiu, C.-J.; Jou, I.-M.; Chen, C.-Y. Prevalence and associated factors of sarcopenia and severe sarcopenia in older Taiwanese living in rural community: The Tianliao Old People study 04. Geriatr. Gerontol. Int. 2014, 14 (Suppl. 1), 69-75. [CrossRef]

63. Tooth, L.; Bennett, S.; McCluskey, A.; Hoffmann, T.; McKenna, K.; Lovarini, M. Appraising the quality of randomized controlled trials: Inter-rater reliability for the OTseeker evidence database. J. Eval. Clin. Pract. 2005, 11, 547-555. [CrossRef]

64. Foley, N.C.; Bhogal, S.K.; Teasell, R.W.; Bureau, Y.; Speechley, M.R. Estimates of quality and reliability with the physiotherapy evidence-based database scale to assess the methodology of randomized controlled trials of pharmacological and nonpharmacological interventions. Phys. Ther. 2006, 86, 817-824. [CrossRef]

65. Briani, R.V.; Ferreira, A.S.; Pazzinatto, M.F.; Pappas, E.; De Oliveira Silva, D.; Azevedo, F.M. What interventions can improve quality of life or psychosocial factors of individuals with knee osteoarthritis? A systematic review with meta-analysis of primary outcomes from randomised controlled trials. Br. J. Sports Med. 2018, 52, 1031-1038. [CrossRef]

66. Beaudreuil, J.; Coudreuse, J.M.; Guyen, C.N.; Deat, P.; Chabaud, A.; Pereira, B.; Lorenzo, A.; Sailhan, F.; Rannou, F.; Coudeyre, E. An algorithm to improve knee orthosis prescription for osteoarthritis patients. Ann. Phys. Rehabil. Med. 2016, 59, e156. [CrossRef]

67. Egger, M.; Davey Smith, G.; Schneider, M.; Minder, C. Bias in meta-analysis detected by a simple, graphical test. BMJ 1997, 315, 629-634. [CrossRef]

68. Rosenthal, R. (Ed.) Meta-Analytic Procedures for Social Research; Sage Publications: Newbury Park, CA, USA, 1993.

69. R Core Team. R: A Language and Environment for Statistical Computing; R Foundation for Statistical Computing: Vienna, Austria, 2021.

70. Harrer, M.; Cuijpers, P.; Furukawa, T.A.; Ebert, D.D. Doing Meta-Analysis in R: A Hands-on Guide; PROTECT Lab Erlangen: Erlangen, Germany, 2019.

71. Shim, S.R.; Kim, S.-J.; Lee, J.; Rücker, G. Network meta-analysis: Application and practice using R software. Epidemiol. Health 2019, 41, e2019013. [CrossRef]

72. Higgins, J.P.; Jackson, D.; Barrett, J.K.; Lu, G.; Ades, A.E.; White, I.R. Consistency and inconsistency in network meta-analysis: Concepts and models for multi-arm studies. Res. Synth. Methods. 2012, 3, 98-110. [CrossRef]

73. Jackson, D.; Barrett, J.K.; Rice, S.; White, I.R.; Higgins, J.P. A design-by-treatment interaction model for network meta-analysis with random inconsistency effects. Stat. Med. 2014, 33, 3639-3654. [CrossRef]

74. Rücker, G.; Schwarzer, G. Ranking treatments in frequentist network meta-analysis works without resampling methods. BMC Med. Res. Methodol. 2015, 15, 58. [CrossRef]

75. Mbuagbaw, L.; Rochwerg, B.; Jaeschke, R.; Heels-Andsell, D.; Alhazzani, W.; Thabane, L.; Guyatt, G.H. Approaches to interpreting and choosing the best treatments in network meta-analyses. Syst. Rev. 2017, 6, 79. [CrossRef]

76. Sedgwick, P.; Marston, L. How to read a funnel plot in a meta-analysis. BMJ. 2015, 351, h4718. [CrossRef]

77. Caldwell, D.M.; Welton, N.J.; Ades, A.E. Mixed treatment comparison analysis provides internally coherent treatment effect estimates based on overviews of reviews and can reveal inconsistency. J. Clin. Epidemiol. 2010, 63, 875-882. [CrossRef]

78. Reken, S.; Sturtz, S.; Kiefer, C.; Böhler, Y.B.; Wieseler, B. Assumptions of Mixed Treatment Comparisons in Health Technology Assessments-Challenges and Possible Steps for Practical Application. PLoS ONE 2016, 11, e0160712. [CrossRef]

79. Król, P.; Franek, A.; Durmała, J.; Błaszczak, E.; Ficek, K.; Król, B.; Detko, E.; Wnuk, B.; Białek, L.; Taradaj, J. Focused and Radial Shock Wave Therapy in the Treatment of Tennis Elbow: A Pilot Randomised Controlled Study. J. Hum. Kinet. 2015, 47, 127-135. [CrossRef]

80. Lohrer, H.; Nauck, T.; Dorn-Lange, N.V.; Scholl, J.; Vester, J.C. Comparison of radial versus focused extracorporeal shock waves in plantar fasciitis using functional measures. Foot Ankle Int. 2010, 31, 1-9. [CrossRef]

81. Sun, J.; Gao, F.; Wang, Y.; Sun, W.; Jiang, B.; Li, Z. Extracorporeal shock wave therapy is effective in treating chronic plantar fasciitis: A meta-analysis of RCTs. Medicine 2017, 96, e6621. [CrossRef]

82. van der Worp, H.; Zwerver, J.; Hamstra, M.; van den Akker-Scheek, I.; Diercks, R.L. No difference in effectiveness between focused and radial shockwave therapy for treating patellar tendinopathy: A randomized controlled trial. Knee Surg. Sports Traumatol. Arthrosc. 2014, 22, 2026-2032. [CrossRef]

83. Zhao, Z.; Ji, H.; Jing, R.; Liu, C.; Wang, M.; Zhai, L.; Bai, X.; Xing, G. Extracorporeal shock-wave therapy reduces progression of knee osteoarthritis in rabbits by reducing nitric oxide level and chondrocyte apoptosis. Arch. Orthop. Trauma. Surg. 2012, 132, 1547-1553. [CrossRef]

84. Cheng, J.H.; Jhan, S.W.; Hsu, C.C.; Chiu, H.W.; Hsu, S.L. Extracorporeal Shockwave Therapy Modulates the Expressions of Proinflammatory Cytokines IL33 and IL17A, and Their Receptors ST2 and IL17RA, within the Articular Cartilage in Early Avascular Necrosis of the Femoral Head in a Rat Model. Mediat. Inflamm. 2021, 2021, 9915877. [CrossRef]

85. Moretti, B.; Iannone, F.; Notarnicola, A.; Lapadula, G.; Moretti, L.; Patella, V.; Garofalo, R. Extracorporeal shock waves down-regulate the expression of interleukin-10 and tumor necrosis factor-alpha in osteoarthritic chondrocytes. BMC Musculoskelet. Disord. 2008, 9, 16. [CrossRef]

86. Liu, B.Y.; Li, H.Y.; Jin, X.Y.; Orthopedics, D. Effect of extracorporeal shock wave therapy on expression of IL-1 $\beta$,TNF- $\alpha$ and MMP-13 in joint fluid for early and middle stage knee osteoarthritis. Orthop. J. Chin. 2016, 24, 1807-1810. 